\title{
Water masers in the Saturnian system
}

\author{
S. V. Pogrebenko ${ }^{1}$, L. I. Gurvits ${ }^{1}$, M. Elitzur ${ }^{2}$, C. B. Cosmovici ${ }^{3}$, I. M. Avruch ${ }^{1,4}$, S. Montebugnoli ${ }^{5}$, E. Salerno ${ }^{5}$, \\ S. Pluchino ${ }^{3,5}$, G. Maccaferri ${ }^{5}$, A. Mujunen ${ }^{6}$, J. Ritakari ${ }^{6}$, J. Wagner ${ }^{6}$, G. Molera ${ }^{6}$, and M. Uunila ${ }^{6}$ \\ 1 Joint Institute for VLBI in Europe, PO Box 2, 7990 AA Dwingeloo, The Netherlands \\ e-mail: [pogrebenko;lgurvits]@jive.nl \\ 2 Department of Physics and Astronomy, University of Kentucky, 600 Rose Street, Lexington, KY 40506-0055, USA \\ e-mail: moshe@pa.uky.edu \\ 3 Istituto Nazionale di Astrofisica (INAF) - Istituto di Fisica dello Spazio Interplanetario (IFSI), via del Fosso del Cavaliere, \\ 00133 Rome, Italy \\ e-mail: cosmo@ifsi-roma.inaf.it \\ 4 Science \& Technology BV, PO 6082600 AP Delft, The Netherlands \\ e-mail: avruch@stcorp.nl \\ 5 Istituto Nazionale di Astrofisica (INAF) - Istituto di Radioastronomia (IRA) - Stazione Radioastronomica di Medicina, \\ via Fiorentina 3508/B, 40059 Medicina (BO), Italy \\ e-mail: [s.montebugnoli;e.salerno;g.maccaferri]@ira.inaf.it; s.pluchino@med.ira.inaf.it \\ ${ }^{6}$ Helsinki University of Technology TKK, Metsähovi Radio Observatory, 02540 Kylmälä, Finland \\ e-mail: [amn; jr;jwagner;gofrito;minttu]@kurp.hut.fi
}

Received 18 October 2008 / Accepted 4 December 2008

\section{ABSTRACT}

\begin{abstract}
Context. The presence of water has long been seen as a key condition for life in planetary environments. The Cassini spacecraft discovered water vapour in the Saturnian system by detecting absorption of UV emission from a background star. Investigating other possible manifestations of water is essential, one of which, provided physical conditions are suitable, is maser emission.

Aims. We report detection of water maser emission at $22 \mathrm{GHz}$ associated with several Kronian satellites using Earth-based radio telescopes.

Methods. We searched for water maser emission in the Saturnian system in an observing campaign using the Metsähovi and Medicina radio telescopes. Spectral data were Doppler-corrected over orbital phase for the Saturnian satellites, yielding detections of water maser emission associated with the moons Hyperion, Titan, Enceladus, and Atlas.

Results. The detection of Saturnian water molecules by remote astronomical observation can be combined with in situ spacecraft measurements to harmonise the physical model of the Saturnian system.
\end{abstract}

Key words. masers - radio lines: solar system - planets and satellites: general

\section{Introduction: feasibility of water maser detection in the Saturian environment}

The Cassini Ultraviolet Imaging Spectrometer (UVIS) detected water vapour in a "plume" emanating from Enceladus by measuring absorption of UV emission from a background star (Hansen et al. 2006). This discovery confirmed Enceladus as a supplier of water to Saturn's ring system and triggered a search for other manifestations of water in the Saturnian (Kronian) system. One of these manifestations is maser emission of water molecules at a frequency of $22 \mathrm{GHz}$ (1.35 cm wavelength). To achieve the maser-amplification optical depth of $\tau>1$, with collisional pumping for $\mathrm{H}_{2} \mathrm{O}$ molecules at a kinetic temperature around $200 \mathrm{~K}$, several requirements should be met (Elitzur 1992; Elitzur et al. 1989, 1992). These requirements can be expressed in terms of a scaling parameter $n_{\mathrm{H}_{2} \mathrm{O}} \cdot n_{\text {collider }} \cdot C \cdot D$, which should exceed a certain threshold. Here $n_{\mathrm{H}_{2} \mathrm{O}}$ and $n_{\text {collider }}$ are volume densities of the water molecules and collider particles, $C$ is the collision rate coefficient, and $D$ is the characteristic size of the maser cloud. In a cloud of size $D \sim 1000 \mathrm{~km}$ with $\mathrm{H}_{2}$ molecules as the dominant collision partner, the threshold requirements for water maser emission are met as described by Elitzur et al. (1989), with $\mathrm{H}_{2}$ and $\mathrm{H}_{2} \mathrm{O}$ number densities of $n_{\mathrm{H}_{2}} \sim 10^{10} \mathrm{~cm}^{-3}$ and $n_{\mathrm{H}_{2} \mathrm{O}} \sim 10^{7} \mathrm{~cm}^{-3}$.

Alternatively, if water is the dominant component, waterwater collisions can ignite maser emission if the number density is $n_{\mathrm{H}_{2} \mathrm{O}} \sim 10^{9} \mathrm{~cm}^{-3}$, under the assumption that the collisional cross-sections of water-water and water-hydrogen do not differ greatly. We note that the precise form of the water-water crosssection is not critical for triggering the inversion. Collisional pumping of the $22 \mathrm{GHz}$ transition was shown to be a robust mechanism, quite independently of the specific excitation crosssection (see Elitzur 1992).

The presence of free electrons with energies below $\sim 0.2 \mathrm{eV}$ and number density $n_{\mathrm{e}} \sim 10^{4}-10^{5} \mathrm{~cm}^{-3}$ relaxes the requirements (Elitzur \& Fuqua 1989), to a cloud of size about $300 \mathrm{~km}$ and water density $n_{\mathrm{H}_{2} \mathrm{O}} \sim 10^{7} \mathrm{~cm}^{-3}$. This low energy electron density is consistent with in situ measurements made by the Cassini spacecraft (Schippers et al. 2008). The amount of water involved in all of the models above is equivalent to an ice cube several tens of metres in size.

At the distance of Saturn $\left(1.3 \times 10^{9} \mathrm{~km}\right)$, a maser cloud of size $\sim 1000 \mathrm{~km}$ and optical depth $\tau \sim 3-10$ in the $22 \mathrm{GHz}$ water line would produce an antenna temperature response of 
$10-40 \mathrm{mK}$ in a $30-\mathrm{m}$ radio telescope. To detect this level of maser signal, an integration time of the order of several hours would be required. Physical models of masers in the Solar System were described by Mumma (1993) and the first detection of the $22 \mathrm{GHz}$ water-maser emission from a planetary system was made during the collision of the Shoemaker-Levy comet with Jupiter (Cosmovici et al. 1996).

\section{Observations and data processing}

We conducted trial observations of the Saturnian system at $22 \mathrm{GHz}$ with the Medicina 32-m radio telescope with a nominal gain of $0.1 \mathrm{~K} / \mathrm{Jy}$ (INAF-IRA, Italy) in April and May 2006, using a Mk5 disk-based VLBI recording system and software correlator-spectrometer developed for the Huygens Probe VLBI tracking experiment (Lebreton et al. 2005). The data were acquired for two circular polarizations, each of $8 \mathrm{MHz}$ bandwidth, centred on the $22235.08 \mathrm{MHz}$ water line shifted in accord with the predicted radial velocity of Saturn (calculated by the NASA JPL Horizons software, Giorgini et al. 1996). This bandwidth corresponded to a $100 \mathrm{~km} \mathrm{~s}^{-1}$ radial velocity span around the maser frequency (compared to a $20 \mathrm{~km} \mathrm{~s}^{-1}$ variation in radial velocity for the inner Saturnian satellites during a typical 8-h observing run). The antenna primary beam of 2 arcmin (FWHP) encompassed all the inner Saturnian rings, part of the E-ring, all the inner satellites, and Dione at certain orbital phases. Four observing runs of $8 \mathrm{~h}$ each were conducted by employing ONsource - OFF-source telescope nodding with a $50 \%$ duty cycle of $6 \mathrm{~min}$. The system temperature of the telescope during these runs was in the range $160 \mathrm{~K}$ to $210 \mathrm{~K}$. This first series of observations indicated the presence of a $\sim 40 \mathrm{mK}$ spectral line (at a $3.5 \sigma$ SNR level) associated with the orbital motion of Dione.

Systematic observations with the Medicina radio telescope commenced in December 2006 with the Direct-FFT Spectrometer (Montebugnoli et al. 1996). The observing setup was basically the same as in the Mk5-based observations, but with one polarization only and an ON/OFF nodding 50\% duty-cycle of $2.7 \mathrm{~min}$. Between December 2006 and December 2007, we compiled about 200 h of observations. In addition, the Metsähovi 14-m radio telescope with a nominal gain of $0.04 \mathrm{~K} / \mathrm{Jy}$ (HUT-TKK, Finland) continued the observing campaign in April 2008, adding another $35 \mathrm{~h}$ in two polarisations with a system noise temperature between $90 \mathrm{~K}$ and $130 \mathrm{~K}$. The Metsähovi data were recorded and processed using a locally-developed software spectrometer.

Data analysis was initiated by applying statistical weighting to the ON-OFF difference spectra for each nodding cycle in accordance with the system temperature, antenna gain (as a function of target elevation), and angular distance between the target and antenna-pointing centre. The spectra were then accumulated, accounting for the Doppler shifts of Saturn and a variety of known Saturnian satellites. In the former case, Doppler tracking was conducted for Saturn's central spot and polar regions (at 0 velocity offset), the equatorial belt (with velocity offsets within $\pm 9 \mathrm{~km} \mathrm{~s}^{-1}$ from 0 ), and ring system (within $\pm 20-25 \mathrm{~km} \mathrm{~s}^{-1}$ velocity offset). For each in-beam Saturnian satellite spectra were accumulated separately for 16 orbital phases to account for a change in the geometry of beamed maser emission. From the total observing time of $235 \mathrm{~h}$, the average ON-source time for each tracked Saturnian satellite in each orbital phase was about $4 \mathrm{~h}$. The average spectra with the velocity range $V_{\text {range }}=50-80 \mathrm{~km} \mathrm{~s}^{-1}$ were then smoothed with a Gaussian kernel of equivalent width $V_{\mathrm{w}}=1 \mathrm{~km} \mathrm{~s}^{-1}$. The resulting spectra had $N_{\mathrm{df}}=V_{\text {range }} / V_{\mathrm{w}}$ degrees of freedom. In the next step, a polynomial of order $N_{\mathrm{p}}$ was removed from the spectra, decreasing the number of degrees of freedom to $N_{\mathrm{df}}-N_{\mathrm{p}}$. The spectra then were searched for any significant signal in a $2.8 \mathrm{~km} \mathrm{~s}^{-1}$ window centred on $0 \mathrm{~km} \mathrm{~s}^{-1}$, i.e. in the rest frame of the target body. Statistical significance was estimated by inflating the rms of the residual baseline-ripple-corrected spectra by the factor $\sqrt{N_{\mathrm{df}} /\left(N_{\mathrm{df}}-N_{\mathrm{p}}\right)}$, accounting for the degrees of freedom removed by polynomial fitting and subtraction.

We realise that our approach of Doppler tracking on the discreet bodies of the Saturnian system does not cover the full range of possible maser emission configurations; the velocity resolution and line width of $\sim 1 \mathrm{~km} \mathrm{~s}^{-1}$ allows us to associate the emission spot with a given Saturnian satellite with a positional accuracy of about $1000 \mathrm{~km}$.

Data processing was conducted for 16 Saturnian satellites, each in sixteen orbital phases, and Saturn itself (obviously, in a single orbital phase). Thus the overall number of output spectra was about 250 . For this number of output spectra, the detection of a spectral feature at the level of $\sim 4 \sigma$ is indicative rather than definitive, while higher levels of detections should be seen as statistically significant.

\section{Results and discussion}

Several bodies in the Saturnian system exhibited significant detections at the expected water maser frequency and one of them (Atlas) had a sufficiently large data set to be tested by splitting it into three independent subsets for analysis. This persistence check gave a positive result.

Based on the signal-to-noise ratio (SNR) of the potential detections, from the total of 20 objects in the Saturnian system for which data had been analysed, we selected four candidates for further investigation: Titan, Hyperion, Atlas, and Enceladus. The typical detections are at the level of $\sim 30-50 \mathrm{mK}$ for Medicina and $\sim 20 \mathrm{mK}$ for Metsähovi, which translate into flux densities of $0.3 \mathrm{Jy}$ to $0.5 \mathrm{Jy}$ using the nominal antenna gains of the telescopes. An additional uncertainty of $30 \%$ due to various instrumental and propagation fluctuations cannot be discounted. No other objects among the investigated targets showed a detection level exceeding $3.5 \sigma$.

Water maser detections for Titan and Hyperion are summarised in Fig. 1, which indicates the phases during which data were obtained and the final spectra for orbital phases six and eight, which exhibit the strongest indications of emission. These spectra resulted from total integration times of six hours on Hyperion and eight hours on Titan. The fact that the strongest detections correspond to spatially coincident phases for both satellites might be indicative of a common cause of maser excitation, e.g. proximity to the Saturnian magnetospheric bow shock.

Figure 2 presents spectra reduced for the orbital motion of Atlas, the most secure detection of our observing campaign. Phase 5, which has the highest SNR detection $(6.5 \sigma)$, corresponds to the orbital segment shown in red in the upper left panel of Fig. 2. The high SNR of this detection and its persistence over one year of observation (as illustrated on the right panel of Fig. 2) allowed us to associate the maser emission with a spot lagging the position of Atlas by several thousand $\mathrm{km}$ along its orbit rather than Atlas itself, suggesting that emission can originate in the edge regions of rings A and F, disturbed by the Atlas's motion.

A $4.2 \sigma$ detection of water maser toward Enceladus by Metsähovi is presented in Fig. 3. The water vapor column and volume densities implied by this detection are consistent with 


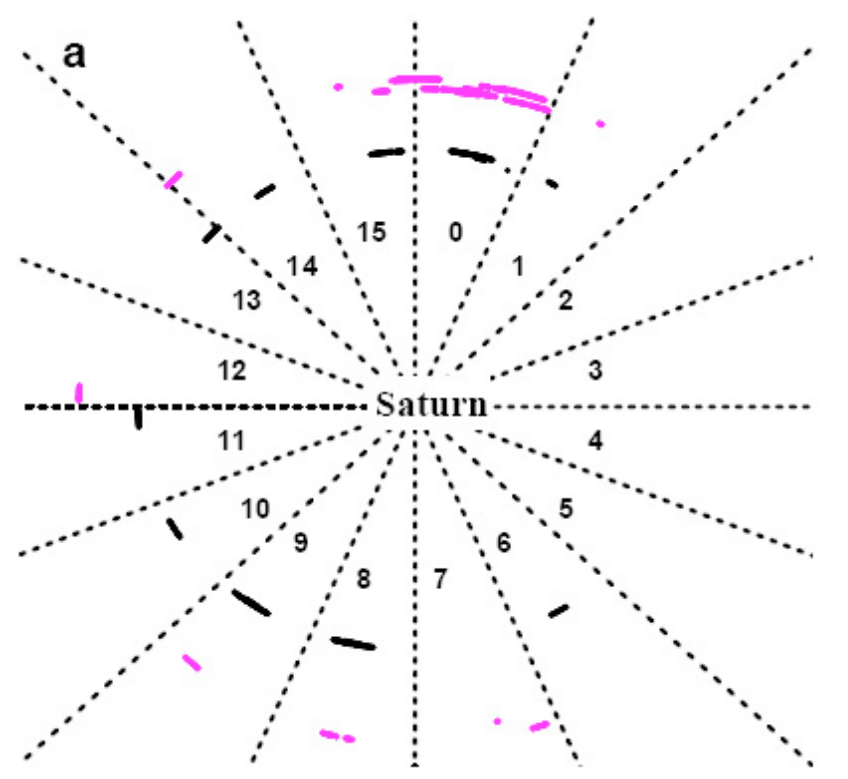

Toward Observer
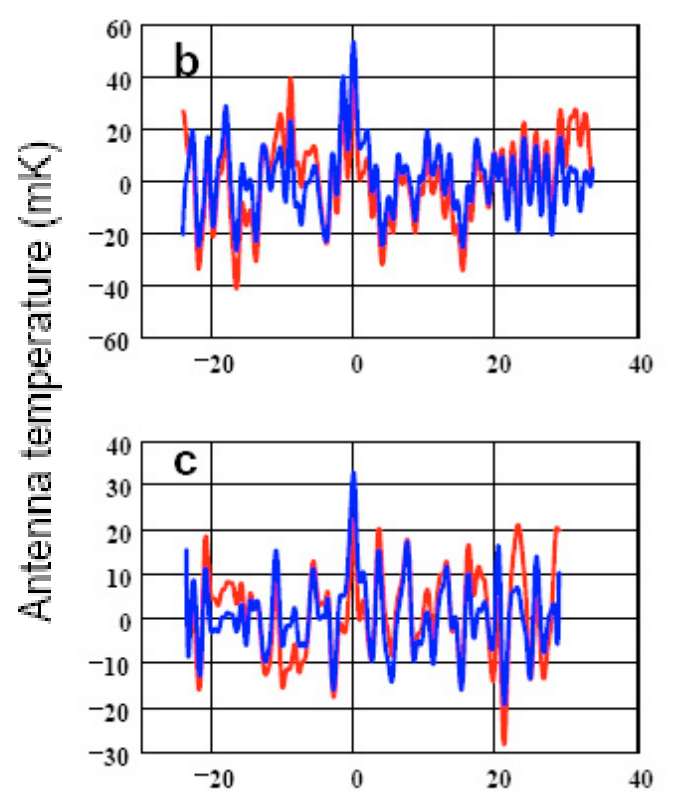

Target-centric velocity offset $(\mathrm{km} / \mathrm{s})$

Fig. 1. Summary plot of Hyperion and Titan detections. a) Distribution of orbital phases for Titan (black) and Hyperion (magenta), for which the data were collected. The observer (Earth) is located along the border between phases 7 and 8. b) The raw Doppler-corrected spectrum (red line) and baseline-fitted spectrum (blue line) for Hyperion orbital phase 8. c) The same for Titan, orbital phases 6 and 8 co-added. The signal-to-noise ratio (SNR) is at the $3.8 \sigma$ level for Titan and $4.0 \sigma$ for Hyperion.
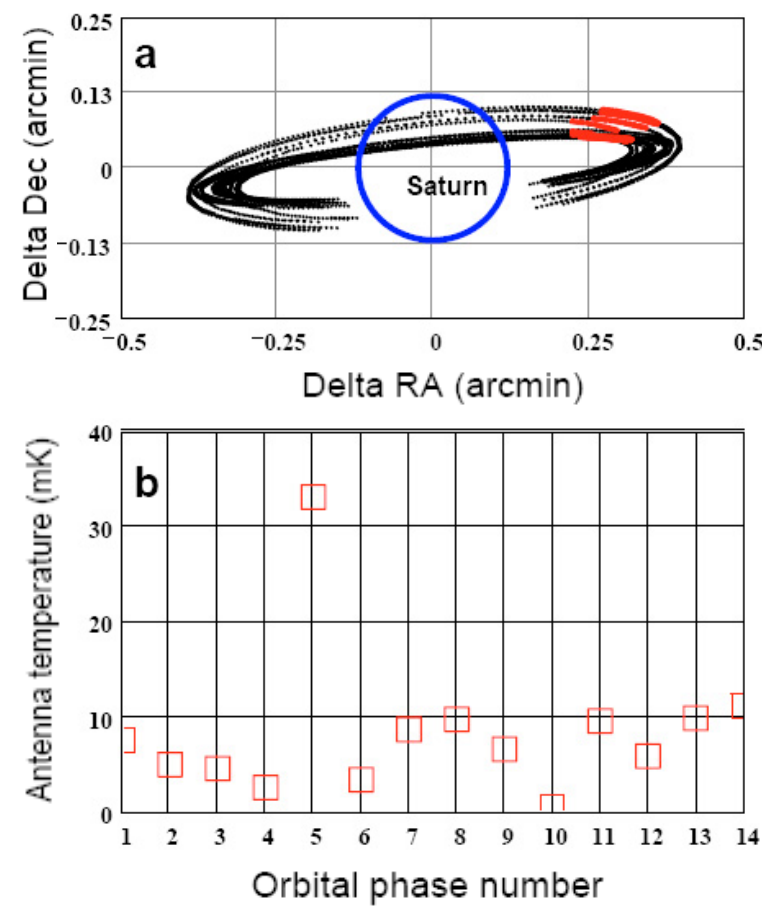

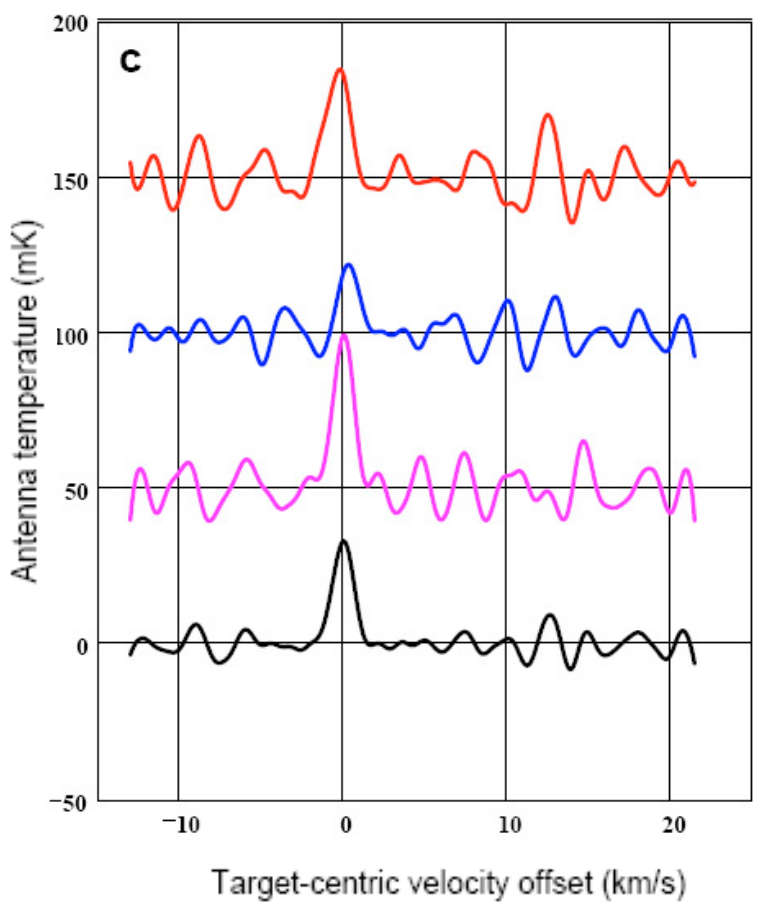

Target-centric velocity offset $(\mathrm{km} / \mathrm{s})$

Fig. 2. Summary plot of Atlas-associated detections. a) Atlas track in delta RA/Dec (arc minutes) with respect to Saturn during our observations black dots. Each dot represents a single 3-min ON+OFF acquisition time. Red dots indicate the Atlas orbital positions from which the emission was detected. They are in an approaching (blue-shifted) phase. Saturn's limb is indicated by a blue circle. b) The antenna temperature at the $0 \mathrm{~km} \mathrm{~s}^{-1}$ target-centric velocity offset for each of the 14 orbital phases processed (phases 0 and 15 were in occultation). c) Spectra. The red, blue and magenta lines represent the spectra integrated for 3 different epochs for Atlas orbital phase 5, about $2 \mathrm{~h}$ integration per each epoch. The black line shows the averaged spectrum with a peak antenna temperature of $32 \mathrm{mK}$ and an SNR at the level of 7.0 $\sigma$.

the detection by Hansen et al. (2006, 2008) of Enceladus' water vapour in UV-absorption. We note that over the preceding 14 months the Medicina telescope did not detect a line exceeding $3 \sigma$ from Enceladus. This might indicate a transient nature of the amount of water, pumping conditions, or beaming geometry, which affects the observability of maser emission.

The amount of experimental data available, and the detections shown in Figs. 1-3, are insufficient to construct a detailed 

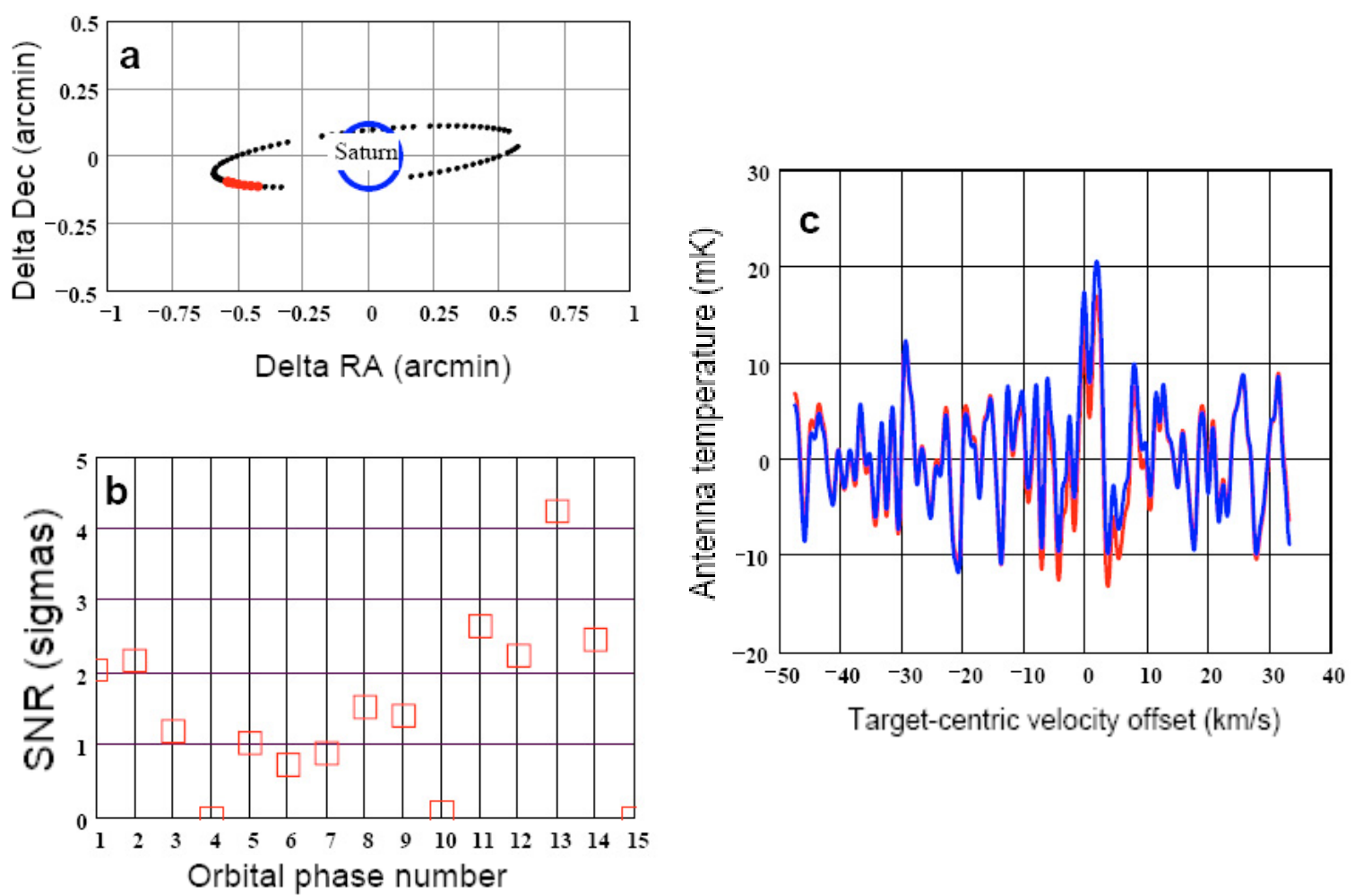

Fig. 3. Summary plot of Enceladus-associated detections. a) Enceladus track in delta RA/Dec (arc minutes) with respect to Saturn during our observations with Metsähovi radio telescope - black dots. Each dot represents a single 20-min ON+OFF acquisition time. Red dots indicate the Enceladus positions from which the emission was detected. Saturn's limb is indicated by a blue circle. b) SNR at the $0 \mathrm{~km} \mathrm{~s}^{-1}$ target-centric velocity offset for each of the orbital phases processed. c) Spectra for the orbital phase with SNR of $4.2 \sigma$; the red line indicates the raw accumulated spectrum, blue line - baseline ripple corrected.

physical model of the maser. In different regions and bodies of the Saturnian system, the maser pumping might vary in cause or character. However, we note that the reasonably edge-on aspect of the planar geometry of the Saturnian system as seen from Earth at the time of these observations is favourable for maser amplification (Elitzur et al. 1992). The strongest detection in our data set, associated with Atlas in the approaching (blueshifted) phase of its orbit (Fig. 2), allows us to roughly estimate the physical parameters in the region of the conjectured water maser. Following Elitzur et al. (1992), and assuming collisions with a neutral molecular agent or low energy electrons to be the main pumping mechanism and a water-vapour ambient temperature in the range of $120-200 \mathrm{~K}$, the required column density of water molecules of $5 \times 10^{13}-5 \times 10^{14} \mathrm{~cm}^{-2}$ will provide a sufficient population inversion to achieve the amplification length $\tau>3$. We note that such a column density is a small fraction of the peak column density of $1.5 \times 10^{16} \mathrm{~cm}^{-2}$ measured by the Cassini UVIS (Hansen et al. 2006, 2008) for a characteristic length of $\sim 100 \mathrm{~km}$. Other pumping mechanisms, such as interaction with the Saturnian magnetosphere, solar-wind plasma and shocks, also cannot be excluded.

\section{Conclusions}

We consider the results presented here as being indicative of the existence of water vapour in the Saturnian system under physical conditions suitable for the generation of maser emission at $22 \mathrm{GHz}$. The possibility of a water vapour maser in the Solar System is particularly attractive because it offers a rare opportunity in astrophysics to study the same phenomenon by means of both radio astronomical observations and in situ measurements. A variety of physical processes capable of exciting masing of water molecules are consistent with conditions in the Saturnian environment. Differentiating between the possible pumping mechanisms using further observations by new, advanced radio telescopes could yield detailed diagnostics of the physical conditions in the Saturnian system.

Acknowledgements. We are grateful to C. J. Hansen, J.-P. Lebreton, B. Magee, M. Perry, D. E. Shemansky and J. H. Waite Jr. for advice and stimulating discussions, E. Flamini for persistent support, and the anonymous referee for useful suggestions. The work at JIVE was partially supported by the ESA-ESTEC Contract No. 18386, and at INAF-IRA by the Italian Space Agency (ASI) contracts I/R/059/04 and I/023/06/0. M.E. acknowledges the NSF grant AST-0507421.

\section{References}

Cosmovici, C. B., Montebugnoli, S., Orfei, A., Pogrebenko, S., \& Colom, P. 1996, Planet. Space Sci., 44, 735

Elitzur, M. 1992, Astronomical Masers (Dordrecht: Kluwer Academic Publishers)

Elitzur, M., \& Fuqua, J. B. 1989, ApJ, 347, L35

Elitzur, M., Hollenbach, D. J., \& McKee, C. F. 1989, ApJ, 346, 983

Elitzur, M., Hollenbach, D. J., \& McKee, C. F. 1992, ApJ, 394, 221

Giorgini, J. D., Yeomans, D. K., Chamberlin, A. B., et al. 1996, BAAS, 28(3), 1158; also http: //ssd.jpl .nasa.gov/horizons.cgi

Hansen, C. J., Esposito, L., Stewart, A. I. F., et al. 2006, Science, 311, 1422

Hansen, C. J., Esposito, L., Stewart, A. I. F., et al. 2008, Nature, 456, 477

Lebreton, J.-P., Witasse, O., Sollazzo, C., et al. 2005, Nature, 438, 758

Montebugnoli, S., Bortolotti, C., Buttaccio, S., et al. 1996, Rev. Sci. Instrum., $67(2), 365$

Mumma, M. J. 1993, in Astrophysical Masers, ed. A. W. Clegg, \& G. E. Nedoluha (Berlin: Springer), 455

Schippers, P., Blanc, M., André, N., et al. 2008, J. Geophys. Res., 113, A07208 